\title{
Characterization of a two-component regulatory system from Acinetobacter baumannii that controls biofilm formation and cellular morphology
}

\section{Correspondence \\ Luis A. Actis \\ actisla@muohio.edu}

Received 10 April 2008

Revised 9 July 2008

Accepted 17 July 2008

\author{
Andrew P. Tomaras, † Michael J. Flagler,‡ Caleb W. Dorsey,§ \\ Jennifer A. Gaddy and Luis A. Actis
}

Department of Microbiology, Miami University, Oxford, OH 45056, USA

\begin{abstract}
Acinetobacter baumannii forms biofilms on abiotic surfaces, a phenotype that may explain its ability to survive in nosocomial environments and to cause device-related infections in compromised patients. The biofilm proficiency of the 19606 type strain depends on the production of pili, cell-surface appendages assembled via the CsuAB-A-B-C-D-E chaperoneusher secretion system. The screening of a bank of isogenic insertion derivatives led to the identification of a biofilm-deficient derivative in which a transposon insertion disrupted a gene predicted to encode the response regulator of a two-component regulatory system. This gene, which was named bfmR, is required for the expression of the Csu pili chaperone-usher assembly system. This coding region is followed by an ORF encoding a putative sensor kinase that was named bfmS, which plays a less relevant role in biofilm formation when cells are cultured in rich medium. Further examination showed that the $b f m R$ mutant was capable of attaching to abiotic surfaces, although to levels significantly lower than those of the parental strain, when it was cultured in a chemically defined minimal medium. Additionally, the morphology of planktonic cells of this mutant, when grown in minimal medium, was drastically affected, while adherent mutant cells were indistinguishable in shape and size from the parental strain. Together, these results indicate that $\mathrm{BfmR}$ is part of a two-component regulatory system that plays an important role in the morphology of $A$. baumannii 19606 cells and their ability to form biofilms on abiotic surfaces.
\end{abstract}

\section{INTRODUCTION}

Acinetobacter baumannii is a Gram-negative opportunistic human pathogen that has been recognized traditionally as the aetiological agent of severe nosocomial infections in compromised hosts (Bergogne-Berezin, 2001; Joly-Guillou, 2005). More recently, this pathogen has emerged as an important infectious agent among soldiers injured in Iraq and Afghanistan (Davis et al., 2005; Dijkshoorn et al., 2007). A. baumannii is thought to be capable of persisting in the clinical environment by forming biofilms on hospital

tPresent address: Department of Microbiology, University of Colorado Health Sciences Center, Aurora, CO 80045, USA.

łPresent address: Department of Molecular Genetics, Biochemistry, and Microbiology, University of Cincinnati, Cincinnati, OH 45267, USA.

§Present address: Department of Microbiology and Molecular Genetics, Medical College of Wisconsin, Milwaukee, WI 53226, USA.

Abbreviations: HRP, horseradish peroxidase; 5'-RACE, 5' random amplification of cDNA ends; SEM, scanning electron microscopy; TEM, transmission electron microscopy.

The GenBank/EMBL/DDBJ accession number for the 19606 genomic region sequence of Acinetobacter baumannii is AY838282. surfaces and medical devices. This hypothesis has been supported by reports that describe the recovery of this pathogen from abiotic surfaces found in medical devices and environments (Villegas \& Hartstein, 2003). The ubiquity of this pathogen in these environments and its remarkable antibiotic-resistance capabilities (Villers et al., 1998) make it extremely difficult to treat patients infected with this pathogen. It has been reported that A. baumannii forms biofilms on glass (Vidal et al., 1996, 1997) and plastic (Tomaras et al., 2003) surfaces, with the latter process requiring the production and assembly of pili on the cell surface. More recently, it has been shown that a bloodstream isolate of $A$. baumannii produces a protein related to a staphylococcal biofilm-associated protein (Bap), which is also required for the development of biofilms on abiotic surfaces (Loehfelm et al., 2008).

Biofilm formation is controlled by a wide range of environmental signals, which trigger the expression of regulatory processes that fine-tune the expression of cellular functions required to initiate and complete this multi-step cellular process (Stanley \& Lazazzera, 2004). Some of these signals are sensed and transduced by 
two-component regulatory systems (Stanley \& Lazazzera, 2004), which include a membrane sensor kinase that works in conjunction with a response regulator. Genes such as $\mathrm{crc}$ (O'Toole et al., 2000), envZ/ompR (Vidal et al., 1998), cpx (Dorel et al., 1999) and $v s p R$ (Yildiz et al., 2001) are examples of regulatory systems that control biofilm formation by bacteria. In addition, the diverse nature of these regulatory systems reflects the different types of signals and mechanisms that participate in the control of bacterial biofilm development (Schembri et al., 2003). It is possible that $A$. baumannii uses similar regulatory mechanisms to sense environmental signals and control biofilm formation on solid surfaces, such as those made of plastics used in human medicine. To test this hypothesis, we have screened a library of transposon insertion mutants for isogenic derivatives defective in biofilm formation. We show in this report that insertional inactivation of the regulator component of a two-component regulatory system expressed in the A. baumannii 19606 type strain results in biofilm deficiency due to the lack of expression of a chaperone-usher assembly system responsible for the presence of pili on the cell surface. In contrast, the inactivation of the sensor component, encoded by a downstream gene, results in a modest reduction in cell attachment and biofilm formation on plastic. The inactivation of the regulator component also causes a significant alteration in cellular morphology.

\section{METHODS}

Bacterial strains, plasmids, media and culture conditions. The bacterial strains and plasmids used in this work are listed in Table 1. Luria-Bertani (LB) broth (10.0 g tryptone $1^{-1}, 5.0 \mathrm{~g}$ yeast extract $\mathrm{l}^{-1}$ and $5.0 \mathrm{~g} \mathrm{NaCl} \mathrm{l}^{-1}$ ) and agar (LB broth supplemented with $15 \mathrm{~g}$ agar $1^{-1}$ ) were used to maintain all bacterial strains. Tris-M9 minimal medium was used for microscopy experiments and biofilm assays, while phosphate-M9 minimal medium was used only for biofilm assays. The Tris-M9 minimal medium consisted of Tris-M9 salts (12.1 g Tris $1^{-1}, \quad 3.7 \mathrm{~g} \mathrm{KCl} l^{-1}, \quad 1.1 \mathrm{~g} \mathrm{NH}_{4} \mathrm{Cl} \mathrm{l}^{-1}, \quad 0.15 \mathrm{~g}$ $\mathrm{CaCl}_{2} \cdot 2 \mathrm{H}_{2} \mathrm{O} \mathrm{l}^{-1}, 0.14 \mathrm{~g} \mathrm{Na}_{2} \mathrm{SO}_{4} \mathrm{l}^{-1}, 0.1 \mathrm{~g} \mathrm{MgCl}_{2} \cdot 6 \mathrm{H}_{2} \mathrm{O} \mathrm{l}^{-1}$ and $5.0 \mathrm{~g} \mathrm{NaCl}^{-1}$, adjusted to $\left.\mathrm{pH} 7.4\right)$ supplemented with $0.5 \%(\mathrm{w} / \mathrm{v})$

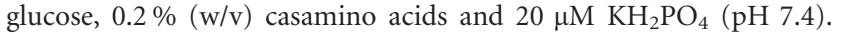
The phosphate-M9 minimal consisted of M9 salts $(6.0 \mathrm{~g}$ $\mathrm{Na}_{2} \mathrm{HPO}_{4} \mathrm{l}^{-1}, \quad 3.0 \mathrm{~g} \quad \mathrm{KH}_{2} \mathrm{PO}_{4} 1^{-1}, \quad 5.0 \mathrm{~g} \mathrm{NaCl} 1^{-1}$ and $1.0 \mathrm{~g}$ $\mathrm{NH}_{4} \mathrm{Cl} \mathrm{l}^{-1}$, adjusted to $\mathrm{pH} 7.4$ ) supplemented with $1.5 \mathrm{mg}$ $\mathrm{CaCl}_{2} \cdot 2 \mathrm{H}_{2} \mathrm{O} \mathrm{l}^{-1}, 12 \mathrm{mg} \mathrm{MgSO} \mathrm{I}^{-1}, 0.5 \%(\mathrm{w} / \mathrm{v})$ glucose and $0.2 \%$ $(\mathrm{w} / \mathrm{v})$ casamino acids. Broth cultures were incubated either with shaking in a rotary shaker at 200 r.p.m. or static at $37^{\circ} \mathrm{C}$. The growth rate of the parental strain and isogenic insertion derivatives in the absence of antibiotics was determined by the $\mathrm{OD}_{600}$ of culture samples taken every hour after inoculation for a period of $24 \mathrm{~h}$. Growth curves were produced twice using fresh overnight inocula each time.

General DNA procedures. Total DNA was isolated either by ultracentrifugation in $\mathrm{CsCl}$ density gradients (Meade et al., 1982) or using a mini-scale method (Barcak et al., 1991). Plasmid DNA was isolated using commercial kits (Qiagen). DNA and Southern blot analyses were conducted using standard protocols (Sambrook \& Russell, 2001). The aph gene from pUC4K (which encodes an

Table 1. Bacterial strains and plasmids used in this study

\begin{tabular}{|c|c|c|}
\hline Strain or plasmid & Relevant characteristics ${ }^{\star}$ & Source or reference \\
\hline 19606 & Clinical isolate, type strain & Bouvet \& Grimont (1986) \\
\hline $\mathrm{Bfm} 273$ & bfmS:: EZ:: TN<R6K $\gamma$ ori/KAN-2> derivative of 19606 & This work \\
\hline \multicolumn{3}{|l|}{ E. coli strains } \\
\hline DH5 $\alpha$ & Used for recombinant DNA methods & Gibco-BRL \\
\hline BL21 Star (DE3) & Protein overexpression strain & Invitrogen \\
\hline BL21 (DE3)pLysS & Protein overexpression strain & Studier et al. (1990) \\
\hline \multicolumn{3}{|l|}{ Plasmids } \\
\hline pCR-Blunt II-TOPO & Used to clone amplicons, $\operatorname{Kan}^{\mathrm{r}}$ & Invitrogen \\
\hline pUC4K & Source of the $\operatorname{Kan}^{\mathrm{r}}$ gene & PharmaciaBiotech \\
\hline pWH1266 & Acinetobacter lwoffii plasmid cloned into pBR322, $\mathrm{Amp}^{\mathrm{r}}, \mathrm{Tet}^{\mathrm{r}}$ & Hunger et al. (1990) \\
\hline pMU360 & bfmR cloned into pET200, $\operatorname{Kan}^{\mathrm{r}}$ & This work \\
\hline pMU515 & $\begin{array}{l}\text { pCR2.1-TOPO derivative used to determine the } b f m R \text { transcription } \\
\text { initiation site }\end{array}$ & This work \\
\hline pMU516 & Plasmid rescued by self-ligation of NdeI-digested DNA from Bfm17 & This work \\
\hline pMU517 & Plasmid rescued by self-ligation of NdeI-digested DNA from Bfm 273 & This work \\
\hline pCWD33 & $\operatorname{csu} A B$ cloned into pGEX4T-2 & This work \\
\hline
\end{tabular}

${ }^{\star} \operatorname{Kan}^{\mathrm{r}}, \mathrm{Amp}^{\mathrm{r}}, \mathrm{Tet}^{\mathrm{r}}$, kanamycin, ampicillin and tetracycline resistant/resistance, respectively. 
aminoglycoside $3^{\prime}$-phosphotransferase responsible for conferring resistance to kanamycin) was used as a $\left[{ }^{32} \mathrm{P}\right] \mathrm{dCTP}$-labelled probe (Feinberg \& Vogelstein, 1983) to detect the insertion of $\mathrm{EZ}: \mathrm{TN}<\mathrm{R} 6 \mathrm{~K} \gamma$ ori/KAN-2 $>$ (Epicentre) as described previously (Dorsey et al., 2002). DNA was sequenced with standard automated DNA sequencing methods using BigDye (Applied Biosystems) chemistry on Applied Biosystems Prism 310 or 3100 instruments. M13 forward and reverse (Yanisch-Perron et al., 1985), T7 and T3 (Invitrogen), or custom-designed primers were used for this purpose. Sequences were assembled using Sequencher 4.2 (Gene Codes Corp.). Nucleotide and amino acid sequences were analysed with DNASTAR, BLAST (http://www.ncbi.nlm.nih.gov), EMBOss (http://liv.bmc.uu.se/ emboss/) and the software available through the ExPASy Molecular Biology Server (http://www.expasy.ch).

Random insertion mutagenesis, rescue of interrupted genes, and genetic complementation of the Bfm17 mutant. A library of 3000 A. baumannii 19606 insertion derivatives, generated with the EZ::TN $<$ R6K $\gamma$ ori/KAN-2>Tnp transposome system as described previously (Dorsey et al., 2002), was screened by careful visual inspection for defects in biofilm formation on microtitre plates stained with crystal violet, as reported previously (Tomaras et al., 2003). The biofilm phenotype of selected derivatives was confirmed with quantitative assays using $3 \mathrm{ml}(7.5 \times 1.1 \mathrm{~cm})$ polystyrene tubes and crystal violet staining (Tomaras et al., 2003). Transposon insertions were confirmed by Southern hybridization of NdeIdigested total DNA with the radiolabelled aph probe. The chromosomal regions harbouring the insertions were rescued using NdeIdigested DNA and sequenced with automated methods. The $b f m R$ parental allele was PCR-amplified using total 19606 DNA as a template, $P f u$ DNA polymerase, and the primers 2065 (5'CGCGGATCCGCGATTGCTTGCATCGTAACG-3') and 2066 (5'CGCGGATCCGCGAATGCAGCAACATCTCCG-3'), both of which included $\mathrm{BamHI}$ restriction sites. The amplicon was ligated into pCRBlunt II TOPO and transformed into Escherichia coli Top10 cells. Plasmid DNA from a kanamycin-resistant transformant was isolated (pMU287) and digested with BamHI, and the fragment was ligated into the cognate site of the A. baumannii-E. coli shuttle vector pWH1266. Plasmid DNA from an ampicillin-resistant, tetracyclinesensitive transformant, named pMU300, was isolated and electroporated into $\mathrm{Bfm} 17$ cells, which were recovered by their resistance to ampicillin $\left(100 \mu \mathrm{g} \mathrm{ml}^{-1}\right)$. The presence and stability of pMU300 in the complemented strain was confirmed by plasmid restriction and Southern blot analyses using BamHI-digested plasmid DNA isolated from five transformants cultured in the presence of ampicillin. The BamHI insert of pMU287 was used to make the $b f m R$ radiolabelled probe for hybridization assays, which were done using standard conditions (Sambrook \& Russell, 2001).

Biofilm assays and electron microscopy. Quantitative biofilm assays of crystal violet-stained cells cultured in either LB or Tris-M9 broth were done as described previously (Tomaras et al., 2003). Duplicate assays were done at least twice using fresh samples each time and representative experiments are reported. For transmission electron microscopy (TEM), cells grown on Tris-M9 agar plates were lifted onto carbon-coated grids, stained with ammonium molybdate and visualized as described previously (Tomaras et al., 2003). For scanning electron microscopy (SEM), broth cultures were grown in square Petri dishes as described above, after which $1 \mathrm{ml}$ of culture (representing planktonic-phase cells) was transferred to a plastic tube, and the rest of the plate (containing sessile cells) was rinsed vigorously with distilled water. Both sets of samples were then fixed as described previously (Tomaras et al., 2003). After fixation, square pieces $(1 \times 1 \mathrm{~cm})$ were cut from the sides of the plates with a small clean wire cutter. These pieces, as well as the planktonic cell samples, were processed and visualized using SEM, as described previously (Tomaras et al., 2003).
Transcriptional and regulatory analysis of the bfmRS locus. Total RNA was isolated from the parental 19606 strain and the Bfm17 and Bfm273 mutants as described previously (Wu \& Janssen, 1997), and each RNA sample was further purified using the Qiagen RNeasy Total RNA Isolation System including the RNase-free DNase incolumn treatment, before amplification using one-step RT-PCR (Qiagen). The primers 1978 (5'-TACGTGGTATCGAATACG-3') and 2036 (5'-ATTGCTTGCATCGTAACG-3') (Fig. 2a) were used to amplify the $b f m R-b f m S$ intergenic region. The primers 1982 (5'-CGTATGCATCAGGTCGAC-3') and 2811 (5'-ACAGACAAAAGCCTGCC- $3^{\prime}$ ) (Fig. 2a) were used to amplify an internal bfmS region. Transcription of the csu locus was tested by RT-PCR using RNA isolated from parental and Bfm17 cells either harbouring no plasmid or electroporated with pMU300 as template and the primers $1662\left(5^{\prime}\right.$ TTACTGGTCAGGTTGACG-3') and 1035 (5'-ACCAGCACACTCGATCTG-3'), which were designed to amplify the $\operatorname{csuAB-csuA}$ intergenic region (Tomaras et al., 2003). The amplicons were analysed by $0.8 \%$ agarose gel electrophoresis using the Tris-acetate/EDTA buffer system and ethidium bromide staining (Sambrook \& Russell, 2001). The identity of the amplicons was confirmed by standard automated capillary DNA sequencing. DNA contamination of the RNA samples was tested by PCR using Pfu DNA polymerase, the appropriate set of primers and total RNA without reverse transcriptase as a template.

The $b f m R$ promoter and transcription initiation site were predicted using the prokaryotic tool of the Neural Network Promoter Prediction program (http://www.fruitfly.org/seq_tools/promoter. html). Promoter elements were mapped with the $5^{\prime}$ Rapid Amplification of cDNA Ends ( $5^{\prime}$-RACE) system from Invitrogen. A. baumannii 19606 RNA isolated from a culture grown in LB broth with shaking was reverse-transcribed with the primer $2810\left(5^{\prime}\right.$ AATGTTGTGGGTCGTCAC-3'). The cDNA was TdT-tailed and PCR-amplified using the gene-specific primer 2036 (5'ATTGCTTGCATCGTAACG-3') and the abridged anchor primer supplied with the kit. The PCR mixture was diluted $1: 100$ and $5 \mu \mathrm{l}$ was used in a subsequent $50 \mu \mathrm{l}$ amplification reaction using the nested gene-specific primer 2037 (5'-CGTTTGATGCAAGCAACC$\left.3^{\prime}\right)$ and the universal amplification primer supplied with the kit. The $590 \mathrm{bp}$ amplicon was recovered from an agarose gel using the QIAEX II gel extraction kit (Qiagen) and was either sequenced using the nested gene-specific primer 2037 or cloned using the Invitrogen TOPO TA cloning kit. Plasmid DNA (pMU515) isolated from a single colony was used as a template to determine the nucleotide sequence of the insert with the M13 (Yanisch-Perron et al., 1985) and T7 primers.

Overexpression, purification and generation of anti-BfmR and anti-CsuAB antibodies. $b f m R$ was PCR-amplified using 19606 total DNA, Pfu DNA polymerase, and the primers 2277 (5'-CACCATGAGCCAAGAAGAAAAG-3') and 2278 (5'-GACCAACCTTATAGGAAG- $\left.3^{\prime}\right)$. The amplicon was ligated into pET200 and transformed into E. coli Top10. Plasmid DNA from a kanamycin-resistant colony (pMU360) was isolated, sequenced with the T7 forward and reverse primers provided with the kit (Invitrogen) to verify the proper orientation of the insert, and transformed into BL21 Star. A transformant was used to overexpress the His-tagged protein, which was isolated by $\mathrm{Ni}^{2+}$ affinity column chromatography from cell lysates prepared as recommended by the manufacturer's protocol (Pierce). Rabbit antibodies against the purified His-tagged BfmR derivative were raised and absorbed using the $\mathrm{Bfm} 17$ mutant strain, as described previously (Actis et al., 1995).

The csuAB-coding region was PCR amplified using Pfu DNA polymerase and the primers $5^{\prime}$-CsuAB-BamHI (5' -CGGGATCCGCTGTTACTGGTCAGGTTGACG-3') and 3'-CsuAB-EcoRI (5'-GGAATTCATAATCGCCTTGTGCTTTTGGTG-3'), which included the 
respective restriction sites at each end of the amplicon. This amplicon was ligated to BamHI- and EcoRI-digested pGEX4T-2 (Amersham Biosciences) and the resulting plasmid, pCWD33, was transformed into E. coli BL21 cells. The CsuAB protein was overexpressed and purified as described for MisL from Salmonella enterica (Dorsey et al., 2005). Rabbit antiserum against the purified GST-CsuAB fusion protein was raised and adsorbed with IPTG-induced E. coli BL21 cells harbouring the empty pGEX4T-2 vector.

Detection of BfmR and CsuAB. LB broth overnight cultures were used to prepare whole-cell lysates as well as total and outermembrane fractions, as described previously (Actis et al., 1985; Dorsey et al., 2003). Equal amounts of total cell lysates and proteins were size-fractionated on $12.5 \%$ polyacrylamide gels (Actis et al., 1985), transferred to nitrocellulose (Towbin et al., 1979), and incubated with either anti-BfmR or anti-CsuAB antibodies. The immunocomplexes were detected by chemiluminescence using horseradish peroxidase (HRP)-labelled protein A. Protein concentrations were determined as described previously (Bradford, 1976).

\section{RESULTS AND DISCUSSION}

\section{Identification of a polystyrene attachment- defective mutant of A. baumannii 19606}

Screening of 3000 derivatives of an A. baumannii 19606 EZ: : TN $<$ R6K $\gamma$ ori/KAN-2 $>$ Tnp transposome insertion library in 96-well microtitre plates with crystal violet assays resulted in the identification of four derivatives unable to form biofilms and eight clones that showed an appreciable reduction but not abolition of biofilm formation. Bfm17, one of the biofilm-null mutants, showed no detectable attachment to or biofilm formation on $3 \mathrm{ml}$ polystyrene tubes stained with crystal violet when compared with the parental strain (Fig. 1a). The transposon insertion was mapped by rescue cloning and DNA sequencing within an ORF predicted to code for a 238 aa protein, whose amino acid sequence is significantly related (E values higher than $1 \times \mathrm{e}^{-5}$ ) to a large number of predicted and bona fide bacterial response regulators when analysed using BLASTX and the GenBank database. Genetic complementation of the Bfm17 mutant with pMU300, a derivative of pWH1266 that contains a cloned copy of the parental coding region (Table 1), restored biofilm formation to levels similar to those of the parental strain. In contrast, Bfm17 cells harbouring the empty pWH1266 vector showed no detectable attachment (Fig. 1a). Although not shown, Southern blot hybridization proved that the Bfm 17 derivative has a single transposon insertion, which maps within a $13.8 \mathrm{~kb}$ NdeI restriction fragment that harbours the coding region identified by insertion mutagenesis. Restriction and DNA hybridization analyses showed that all $\mathrm{Bfm} 17$ complemented derivatives examined harboured pMU300 within a $1.7 \mathrm{~kb}$ BamHI insert and without detectable DNA rearrangements. Furthermore, the insertion inactivation of $b f m R$ and the complementation of Bfm17 with pMU300 did not result in significant differences in the growth rate of cells cultured in either LB or Tris-M9 broth when compared with that displayed by the 19606 parental cells (data not shown). (a)

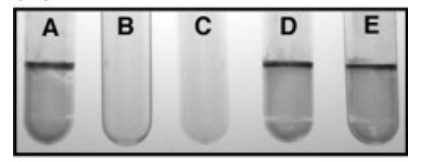

(b)

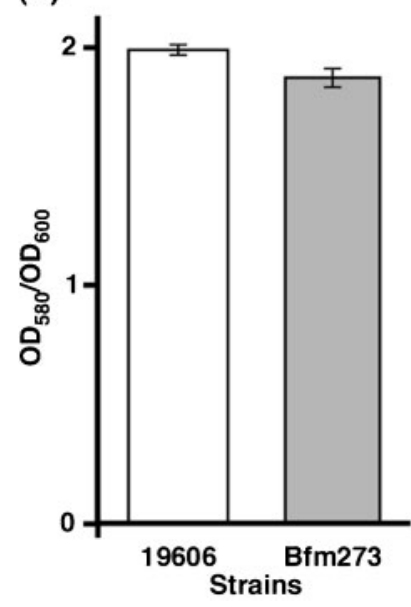

Fig. 1. Detection of biofilms formed on $3 \mathrm{ml}$ plastic tubes. (a) Qualitative biofilm assay of the A. baumannii 19606 parental strain $(\mathrm{A})$, and the isogenic derivatives $\mathrm{Bfm} 17$ (B), Bfm17 harbouring pWH1266 (C), Bfm17 harbouring pMU300 (D) and Bfm273 (E). Bacterial cells were grown without shaking in $3 \mathrm{ml}$ polystyrene tubes in LB broth and stained with crystal violet. (b) Quantitative biofilm assays of 19606 and Bfm273 cells grown as described above, stained with crystal violet and processed as described previously (Tomaras et al., 2003). Error bars, 1 SD from one representative experiment.

The same library screening and confirmation processes resulted in the isolation of $\mathrm{Bfm} 273$, a derivative that displayed a noticeable but not drastic defect in biofilm formation on microtitre plates and plastic tubes (Fig. 1a). Accordingly, quantitative biofilm assays showed that this mutant has a slight but distinguishable defect in its ability to form biofilms on polystyrene tubes when compared with the parental strain (Fig. 1b). Rescue cloning, DNA sequencing and Southern blot hybridization using the aph probe showed that the insertion of the transposon in Bfm 273 was located in a $7.2 \mathrm{kbp} \mathrm{NdeI} \mathrm{restriction} \mathrm{fragment}$ different from that harbouring the transposon in the Bfm17 mutant (data not shown). This insertion derivative and the parental strain 19606 did not show significant differences in growth rate when cultured in LB or Tris-M9 broth.

\section{Sequence analysis of the Bfm17 and Bfm273 insertions}

DNA sequencing of the NdeI-rescued fragments harbouring the transposon insertion in the Bfm17 and Bfm 273 
(a)

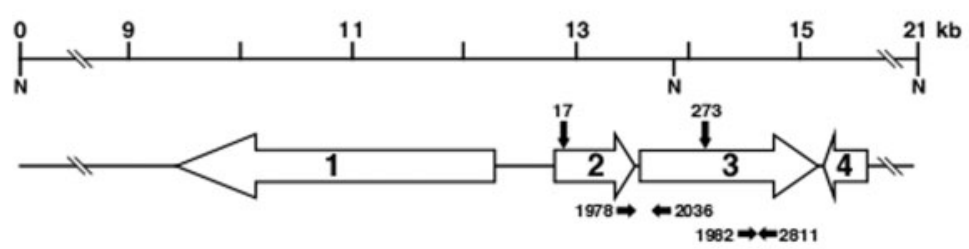

(b)

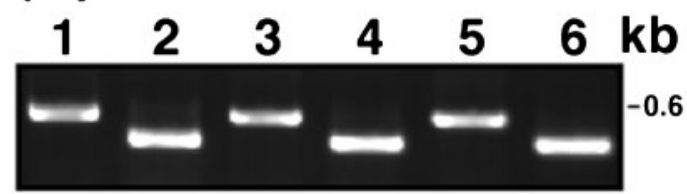

(c)

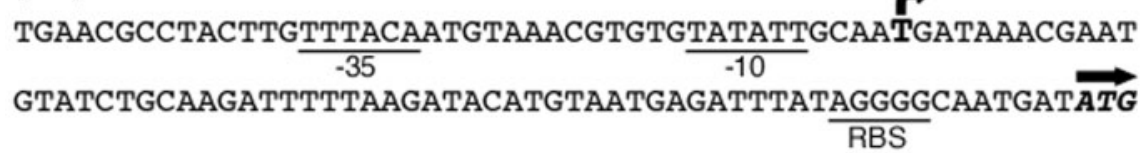

Fig. 2. Analysis of the bfmRS locus. (a) Genetic organization of the chromosomal region harbouring the bfmRS genes (ORFs 2 and 3 , respectively) flanked by ORFs 1 and 4 encoding the ribonucleotide reductase $\alpha$ subunit ( $n r d A$ ) and a conserved hypothetical protein, respectively. ORFs are represented according to their chromosomal location and direction of transcription. The vertical arrows indicate the transposon insertion in the 17 and 273 derivatives. The vertical lines underneath the horizontal line shown on the top portion of this panel indicate the location of the $N$ del $(\mathrm{N})$ restriction sites. Custom-designed primers used in RT-PCR experiments are shown as black horizontal arrows with their corresponding numbers. (b) RT-PCR analysis of total RNA isolated from A. baumannii 19606 (lanes 1 and 2), Bfm1 7 (lanes 3 and 4) and Bfm273 (lanes 5 and 6) cells cultured in LB broth. Primers 1978 and 2036 were used to test the co-transcription of both coding regions (lanes 1, 3 and 5), while primers 1982 and 2811 were used to test the transcription of bfmS (lanes 2, 4 and 6). The position of the $0.6 \mathrm{~kb} \lambda$ Hindlll fragment is shown on the right of the figure. (c) bfmRS promoter region. The bfmRS transcription initiation site and the direction of transcription are indicated by the large, bold type and the black curved arrow, respectively. The predicted matching -10 and -35 promoter regions and a putative RBS are also shown. The black horizontal arrow identifies the bfmR initiation codon.

derivatives showed that these two fragments were next to each other. The predicted gene (ORF 1) located immediately upstream of the Bfm17 insertion site (Fig. 2a) encodes a product highly related to the A. baumannii AYE ABAYE3065 $\alpha$ subunit of the ribonucleotide reductase $(n r d A)$. ORF 1 is separated by a $532 \mathrm{nt}$ intergenic region from an oppositely transcribed bicistronic operon harbouring ORFs 2 and 3. This bicistronic operon is followed by a $21 \mathrm{nt}$ intergenic region and a convergently transcribed gene (ORF 4) that encodes a protein highly related to the A. baumannii AYE ABAYE3062 conserved hypothetical protein (http://www.genoscope.cns.fr/agc/mage/baumannoscope). The first ORF of the bicistronic operon (ORF 2, Fig. 2a), which encompasses $714 \mathrm{nt}$ and was interrupted in the Bfm17 biofilm-deficient mutant, encodes a predicted $27.1 \mathrm{kDa}$ cytoplasmic protein with highest similarity to the hypothetical product of the Acinetobacter spp. ADP1 (also known as Acinetobacter baylyi ADP1) ACIAD0726 gene annotated as rstA (http://www.genoscope.cns.fr/agc/mage/ baumannoscope). The list of related sequences generated with BLASTX included sequences found in the A. baumannii
AYE and SDF genomes recently sequenced (Vallenet et al., 2008), as well as a large number of hypothetical and bona fide response regulators consisting of a CheY-like receiver domain and a winged-helix DNA-binding domain. This $A$. baumannii 19606 predicted protein contains the cd00156, cd00383 and COG0745 domains, which are found in classical bacterial response regulators (Martinez-Hackert \& Stock, 1997; Pao \& Saier, 1995; Stock et al., 2000). The cd00156 domain includes a phosphoacceptor site, which is phosphorylated by histidine kinases. cd00383, which represents the effector domain of bacterial response regulators, and the COG0745 domain, which is found in OmpR-like proteins and consists of a CheY-like receiver domain and a winged-helix DNA-binding domain. The two domains of COG0745 are involved in carrying out signal transduction by receiving a phosphate group from the cognate sensor kinase component and binding to promoter elements of downstream target genes (Stock et al., 2000). Based on these similarities, this A. baumannii 19606 gene, which was interrupted in the isogenic derivative $\mathrm{Bfm} 17$, was named $b f m R$. 
The $b f m R$-coding region is followed by ORF 3, which encompasses $1649 \mathrm{nt}$ and is interrupted in the $\mathrm{Bfm} 273$ mutant (Fig. 2a). The product of ORF 3, which starts with a GTG codon, is a predicted 549 aa protein with significant similarity to a large number of bacterial sensor kinases. The predicted product of the A. baumannii SDF gene ABSDF2718 (http://www.genoscope.cns.fr/agc/mage/baumannoscope) is the protein most related to the product of this A. baumannii 19606 ORF. The BLASTX-generated list also included highly related genes identified in the genomes of A. baumannii strains AYE and 17978 and A. baylyi ADP1 (http://www. genoscope.cns.fr/agc/mage/baumannoscope), as well as a large number of predicted as well as genuine sensor histidine kinases found in a wide range of bacteria. Computer analysis of the protein encoded by this A. baumannii 19606 ORF revealed the presence of the pfam00672, cd00082 and cd00075 domains. These conserved sequences represent the HAMP, the histidine kinase A, and the histidine-kinase-like ATPase domains, respectively, which are generally found in bacterial sensor proteins (Stock et al., 2000). The significant similarity to a large number of sensor histidine kinases and the presence of these three domains are consistent with the prediction that this A. baumannii protein is the sensor component of a twocomponent regulatory system. This possibility is supported further by the prediction that this is a cytoplasmic membrane protein that has two transmembrane helices, a feature common to sensor kinases that are part of this particular type of bacterial transcriptional regulation system. Based on these similarities, this A. baumannii 19606 gene, which was interrupted in the isogenic derivative $\mathrm{Bfm} 273$, was named bfmS.

The interruption of $b f m R$ abolished cell attachment and biofilm formation, cell properties that could be restored by complementing the $b f m R$ insertion with the parental $b f m R$ allele alone (Fig. 1a). These observations indicate that the putative response regulator encoded by this gene is essential for cell attachment and initiation of biofilm formation by A. baumannii 19606 cells. In contrast, the interruption of $\mathrm{bfmS}$ produced an appreciable but not drastic reduction in biofilm formation when compared with the parental strain and the $b f m R$ mutant under the same experimental conditions. This result indicates that $\mathrm{BfmS}$ is not essential for biofilm regulation and that alternative sensing pathways can activate $\mathrm{BfmR}$ and control the expression of downstream target genes, as has been described for other cellular processes such as chemotaxis, sporulation and quorum sensing (Bourret \& Stock, 2002; Jiang et al., 2000; Mok et al., 2003). This possibility is consistent with the hypothesis that bacterial cells sense different environmental signals and use different signal transduction pathways that ultimately result in the activation or repression of target genes involved in biofilm development (Stanley \& Lazazzera, 2004). Another possibility is that BfmR is activated by non-cognate sensor kinases, as has been described for the interaction between the Bacillus subtilis YycFG and PhoPR systems (Howell et al., 2006), which are part of a signalling network that senses and controls gene expression in response to changes in phosphate concentration, or even the low-molecularmass phosphodonor acetyl phosphate, in a process known as 'cross-talk' (Wanner, 1992). While this alternate sensing pathway model is plausible, another equally plausible model is that BfmR may be active to stimulate biofilm formation in the non-phosphorylated but not in the phosphorylated state. This possibility is supported by the mechanism of action of the B. subtilis DegS/DegU regulatory system, in which the non-phosphorylated form of the response regulator DegU activates genetic competence while its phosphorylation by the kinase DegS abolishes such a genetic activation (Dahl et al., 1992).

\section{Transcriptional and translational analyses of the bfmRS operon}

A 32 bp intergenic region with no obvious transcription termination signals separates these two coding regions, which could be co-transcribed from a promoter element located upstream of $b f m R$. Such a possibility was confirmed by the production of the predicted $519 \mathrm{bp}$ amplicon (Fig. 2b) when RNA isolated from the 19606 parental strain was tested with RT-PCR using the primers 1978 and 2036 (Fig. 2a). Furthermore, RT-PCR analysis of RNA isolated from the Bfm17 mutant with the primers 1982 and 2811 resulted in the predicted 341 bp replicon internal to $b \mathrm{fmS}$ (Fig. 2b). The same amplicons were produced when total RNA isolated from $\mathrm{Bfm} 17$ and Bfm273 cells cultured in LB was used as a template for RT-PCR analysis using the aforementioned sets of primers (Fig. 2b). Altogether, these results indicate that the insertions in the $b f m R$ and $b f m S$ mutants neither stopped transcription of interrupted genes nor produced polar effects on co-transcribed genes.

Computer analysis of the $b f m R$ immediate upstream region showed the presence of potential transcriptional initiation signals and promoter elements, predictions that were confirmed by determining the transcription initiation site using the 5'-RACE system and 19606 total RNA isolated from cells cultured in LB broth. This approach showed that the C-tailing of the $590 \mathrm{bp}$ amplicon mapped the transcriptional initiation site to the $\mathrm{T}$ located $64 \mathrm{bp}$ upstream of the $b f m R$ initiation codon (Fig. 2c). Based on this observation, we were able to identify putative -10 and -35 promoter sequences (Fig. 2c), each of which had only one mismatch with the $E$. coli consensus sequence for each of these promoter elements. Similarly, a potential RBS that resembles the consensus sequence defined for this translation signal (Shine \& Dalgarno, 1974) was located 7 nt upstream of the $b f m R$ initiation codon (Fig. 2c). It is possible that the initiation site of the $b f m R S$ transcript differs from that shown in Fig. 2(c) when cells are cultured in a different medium, such as the Tris-M9 broth we have used in this work. If that were to be the case, it would indicate that different $b f m R$ transcriptional products could play different regulatory roles, which could affect different 
cellular processes depending on the conditions under which cells are cultured.

Western blotting of A. baumannii 19606 total cell lysates with anti-BfmR polyclonal antibodies resulted in the detection of a single $27 \mathrm{kDa}$ protein band, which was not detected in the cell lysate of the Bfm17 biofilm-deficient derivative (Fig. 3). Electroporation of Bfm17 cells with pMU300 restored the synthesis of this protein, which appeared to be produced in larger amounts when compared with that detected in the parental strain (Fig. $3)$. This result is consistent with the observation that biofilm formation by $\mathrm{Bfm} 17$ can be restored upon the electroporation of a plasmid containing a wild-type copy of $b f m R$ (Fig. 1a). The increased BfmR production in the complemented mutant is most probably due to the higher copy number of the wild-type $b f m R$ allele in this derivative than in the 19606 parental strain. Analysis of different subcellular fractions showed that most of the BfmR was in the cytoplasmic protein fraction of A. baumannii 19606 cells cultured in LB broth (Fig. 3), a location that was not affected by culturing cells in Tris-M9 broth (data not shown). These results indicate that $\mathrm{BfmR}$, which is a predicted response regulator that could transduce environmental signals and control biofilm formation, is a cytoplasmic protein that is required for A. baumannii 19606 to attach to and form biofilms on plastic surfaces.

\section{Electron microscopy and csuAB expression analyses}

Based on the observation that pili assembly is required for biofilm formation by A. baumannii 19606 (Tomaras et al., 2003) and the fact that the Bfm 17 insertion derivative does not make biofilms, we hypothesized that BfmR controls the expression of the csu operon and the assembly of pili. TEM analysis of cells lifted from a Tris-M9 minimal medium agar plate showed that, when compared with the parental

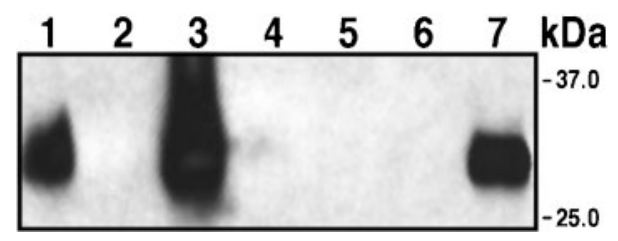

Fig. 3. Detection of BfmR in bacterial cell lysates and subcellular fractions. Whole-cell lysates were prepared from 19606 parental (lane 1) and Bfm17 cells either harbouring no plasmid (lane 2) or electroporated with pMU300 (lane 3) and cultured in LB broth. Total membrane (lane 4), outer-membrane (lane 5), innermembrane (lane 6) and cytoplasmic (lane 7) fractions were isolated from the 19606 parental strain. The blotted proteins were probed with anti-BfmR serum and the immunocomplexes were detected by chemiluminescence using HRP-labelled protein A. The mobility of molecular mass markers is shown to the right of the figure. strain, the Bfm17 mutant lacks any detectable surface pili (Fig. 4). In contrast, complementation of the mutant with the parental $b f m R$ allele cloned in pWH1266 (pMU300) restored pili production, although at lower levels when compared with parental cells (compare panels a and $c$ of Fig. 4). This observation could be due to the overexpression of the $\operatorname{csu} A B-A-B-C-D-E$ operon caused by higher $\mathrm{BfmR}$ intracellular concentration in this derivative (Fig. 3, lane 3). Such an outcome could alter the pili assembly process in the BfmR-complemented strain because of the potentially unbalanced overproduction of some of the pili assembly components. Nevertheless, the restoration of pili formation in the complemented $\mathrm{Bfm} 17$ mutant correlates well with its ability to form biofilms on polystyrene in a fashion similar to that displayed by the parental strain (Fig. 1a).

We also showed the importance of the products of the $\operatorname{csu} A B-A-B-C-D-E$ polycistronic gene cluster in the production and assembly of pili, as well as in the subsequent formation of biofilms by A. baumannii 19606 (Tomaras et al., 2003). Because the phenotype of the Bfm 17 mutant was non-piliated, we investigated whether the expression of the $c s u$ genes was affected by the disruption of $b f m R$ in the insertion derivative $B f m 17$. We tested the expression of $\operatorname{csu} A B$ because it is the first ORF of this gene cluster that appears to encode the major subunit of the pili produced by this assembly system (Tomaras et al., 2003). As shown in Fig. 5(a), RT-PCR analysis of the $c s u A B-c s u A$ intergenic region revealed that RNA from the $\mathrm{Bfm} 17$ mutant failed to yield the product detected when RNA isolated from the parental strain was used as a template. RT-PCR analysis of RNA isolated from Bfm17 cells harbouring pMU300 showed that the complementation of this mutant with the $b f m R$ parental allele was enough to restore transcription of the csu locus. Further expression analysis using an anti-CsuAB serum showed the presence of the CsuAB protein in the whole-cell lysate of the parental strain cultured in either LB or Tris-M9 broth (Fig. 5b, lanes 1 and 2 ). This protein band was not detected in planktonic Bfm17 cells either cultured in LB or Tris-M9 broth or collected from biofilms formed on plastic after incubation in the latter medium (Fig. 5b, lanes 3-5). In contrast, the production of $\mathrm{CsuAB}$ in the $\mathrm{Bfm} 17$ mutant was restored when complemented with pMU300 (lane 6 of Fig. 5b). This analysis also showed that the disruption of $b f m S$ in the Bfm273 insertion derivative did not abolish the production of CsuAB (Fig. 5b, lane 7), an observation that is in accordance with the biofilm phenotype of the Bfm 273 insertion derivative and the dispensable role that $\mathrm{BfmS}$ seems to play in the control of biofilm formation on plastic surfaces. Ponceau $S$ staining of the protein blot before probing with anti-BfmR serum proved that the differences in band intensities shown in the figure are due to variations in protein loading rather than differential production in different culture media.

From these results it is possible to conclude that $\mathrm{BfmR}$ is a transcriptional activator that controls the expression of the 

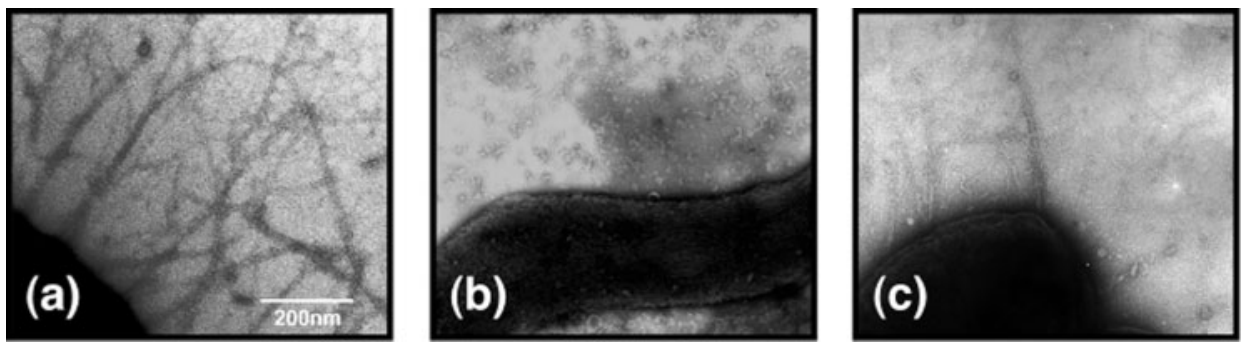

Fig. 4. TEM analysis of $A$. baumannii 19606 and isogenic insertion-derivative cells cultured on Tris-M9 agar and stained with ammonium molybdate. Micrographs showing (a) parental strain 19606, (b) Bfm17 insertion derivative and (c) Bfm17 harbouring pMU300 are at the same magnification.

csu operon, which is required for pili assembly, cell attachment and biofilm formation on abiotic surfaces such as those of polystyrene plates and tubes. This conclusion is also in agreement with the in silico results, which predict that the product of $b f m R$ is the response regulator of a twocomponent regulatory system related to a large number of bacterial proteins that activate the transcription of downstream target genes. Based on these data, it is also

\section{(a)}

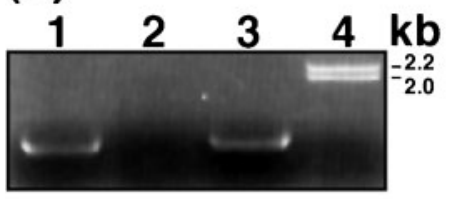

(b)

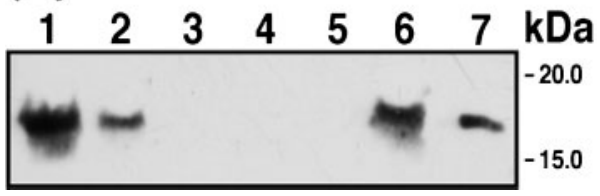

Fig. 5. csuAB expression analysis. (a) Detection of csuAB transcripts by RT-PCR of total RNA isolated from the 19606 parental strain (lane 1) and the Bfm17 insertion derivative either harbouring no plasmid (lane 2) or electroporated with pMU300 (lane 3). Lane 4, Hindlll-digested $\lambda$ DNA size markers. (b) Detection of CsuAB by immunoblotting of total-cell lysates prepared from the 19606 parental strain (lanes 1 and 2), the Bfm17 insertion derivative either harbouring no plasmid (lanes 35) or electroporated with pMU300 (lane 6), and the Bfm273 mutant (lane 7). Planktonic cells used to prepare samples loaded in lanes 1, 3, 6 and 7 were cultured in LB broth, while planktonic cells used to prepare the samples in lanes 2 and 4 were cultured in Tris-M9 broth. Cells collected from biofilms grown in Tris-M9 broth were used to prepare the lysate sample loaded in lane 5 . The mobility of molecular mass markers is shown at the right of the figure. The protein blot was probed with anti-CsuAB serum and the immunocomplexes were detected by chemiluminescence with HRP-labelled protein A. possible to speculate that the BfmRS two-component system could be added to the list of transcriptional regulators already known to be involved in controlling the different steps of bacterial biofilm formation (Stanley \& Lazazzera, 2004). However, the environmental signal or signals that this sensor system recognizes and the target genes that it controls, in addition to $c s u$, remain to be identified. Furthermore, it is not known whether BfmR activates the transcription of the $c s u$ operon via a direct or an indirect pathway, the latter of which could involve a cascade of interacting regulatory signals; these possibilities are being investigated currently.

\section{Media composition affects attachment and biofilm formation}

During these studies we made two additional observations. One of them involves the effect of the culture medium on biofilm formation. As described above, Bfm17 cells cultured in LB broth do not attach to and form detectable biofilms on polystyrene tubes and plates (Fig. 1a). However, crystal violet and light microscopy showed that when the Bfm17 derivative was cultured in Tris-M9 minimal medium, the cells attached to the side of the plates and formed biofilms. As we reported previously (Tomaras et al., 2003), the 19606 parental strain also forms detectable biofilms on polystyrene surfaces when cultured in Tris-M9 minimal medium. Further analysis showed that the biofilm formed by the Bfm 17 cells cultured in LB broth was less than $4 \%$ of that formed by the parental strain under the same experimental conditions, a value that represents the non-specific retention of crystal violet on the tubes without visible cell attachment. In contrast, the amount of biofilm formed by Bfm17 cells was reduced to $39 \%$ of the biofilm formed by the parental strain when cells were incubated in Tris-M9 minimal medium. The electroporation of pMU300 restored the ability of Bfm17 cells to form biofilms to levels similar to or higher than those formed by the parental strain when the cells were incubated in Tris-M9 minimal medium or LB broth, respectively. It is worthy of note that the incubation of $A$. baumannii 19606 in Tris-M9 medium resulted in biofilms 
that were less dense and more patchy than those formed when this strain was cultured in LB broth (data not shown). Similar results were obtained when phosphate-M9 minimal medium was used to culture the parental and Bfm17 cells. These results indicate that the composition of the medium can affect the ability of A. baumannii to form biofilms on abiotic surfaces in a process that appears to be independent of the expression of bfmRS. The $519 \mathrm{bp}$ amplicon produced by RT-PCR of total RNA isolated from 19606 cells cultured in LB broth (Fig. 2b) was also detected when total RNA isolated from biofilm cells cultured in Tris-M9 was used as a template (data not shown). Although not shown, the latter observation is supported by the fact that BfmR was detected in planktonic and biofilm cells cultured in this chemically defined medium. Taken together, these results are also consistent with those of earlier reports which show that the composition of the culture medium has significant effects on biofilm formation. Factors such as the complexity of the media, the presence of different types of carbon sources, and the addition of different amounts of inorganic salts influence the development of bacterial biofilms, as was observed in the case of biofilm formation by Pseudomonas fluorescens (O’Toole \& Kolter, 1998).

The biofilm data reported in the previous paragraph and the observation that the $\mathrm{Bfm} 17$ mutant is a non-piliated derivative that does not express the $c s u$ operon suggest that A. baumannii 19606 could express alternative pili-independent adhesion pathways, which could also lead to biofilm formation on plastic surfaces. An alternative explanation for the capacity of Bfm17 cells to form biofilms when cultured in Tris-M9 minimal medium is that this particular culture medium can induce the expression of the $c s u$ operon and the assembly of pili. However, this does not seem to be the case because immunoblot analysis with anti-CsuAB serum showed that Bfm17 planktonic and biofilm cells grown in this chemically defined medium do not express this pili assembly system (Fig. 5b, lanes 4 and 5).

\section{Role of BfmR in cell morphology}

The other observation made during our studies, which was somewhat unexpected, was that insertional inactivation of $b f m R$ drastically affected the cell morphology of the Bfm 17 isogenic derivative. TEM of cells lifted from colonies formed on the surface of Tris-M9 agar showed that the cells appeared as long filaments with a tendency to aggregate, with very few isolated filaments and almost no cells exhibiting normal morphology (Fig. 6a). The abnormal morphology can be partially appreciated in the micrograph shown in Fig. 4(b), which was taken at a much higher magnification. In addition to piliation, the $\mathrm{Bfm} 17$ cells recovered normal cell morphology after the incorporation by electroporation of pMU300 (Fig. 6b), which harbours a copy of the parental $b f m R$ allele. SEM analysis also showed that planktonic Bfm17 cells, when cultured in Tris-M9 minimal medium, formed long filaments, some of which showed constrictions that appeared to represent the site at which a septum could be formed before cell division (Fig. 6e). In contrast, the parental strain showed the classical cell shape described for this bacterium when cultured under the same experimental conditions (Fig. 6c). The same morphological differences were observed by light microscopy of Gram-stained cells (data not shown). It is important to note that the aberrant morphology of the Bfm17 cells was evident when they were cultured in either Tris-M9 or phosphate-M9 minimal medium, while very few and much shorter filaments were observed when the cells were cultured in LB broth. Furthermore, the aberrant cell morphology, with regard to the number and length of the filaments, was more evident in cells cultured in TrisM9 medium without shaking. It is also important to note that the aberrant cell morphology and the lack of piliation did not result in a clumping phenotype when cells were cultured in LB or Tris-M9 broth.

Fig. 6 shows that the SEM analysis of planktonic and sessile cells obtained from the same Tris-M9 culture sample also revealed that, in contrast to the Bfm 17 planktonic cells (Fig. 6e), the Bfm17 cells attached to the side of the Petri dish (Fig. 6f) had a cell morphology similar to that of the parental strain either in the planktonic (Fig. 6c) or biofilm (Fig. 6d) phase. Similar observations were made when these samples were Gram-stained and examined by standard light microscopy (data not shown). These results indicate that the $\mathrm{BfmR}$ response regulator is also involved in determining the shape of A. baumannii 19606 cells. It is also apparent from these results that contact with and attachment to a plastic surface plays a significant role in the morphology of attached cells by mechanisms that remain to be elucidated.

Currently, we do not know whether the BfmRS regulatory system controls the expression of cellular functions associated with membrane and cell wall synthesis, septa formation and/or cell division. However, there are reports that describe the role of two-component regulatory systems in the morphology of Gram-positive and Gram-negative bacteria. The expression of the MtrAB regulatory system in Corynebacterium glutamicum has a strong effect on cell morphology, osmoprotection and antibiotic susceptibility (Hung et al., 2001). This regulatory system could act by directly controlling cell wall synthesis or osmoregulation, or both processes, which ultimately affect cell morphology. In the case of Streptococcus pneumoniae R6, it has been shown that mutation of the VicRK system results in cell growth, size and morphology defects ( $\mathrm{Ng}$ et al., 2003). These defects appear to be due to the downregulation of $p c s B$, a gene encoding a protein of unknown function that is involved in response to general cell stress. B. subtilis YycFG is another example of a two-component regulatory system that is involved in determining cell shape, although in this case the effect is through the modulation of the ftsAZ operon (Fukuchi et al., 2000). The Fts proteins, particularly FtsZ, are also the targets of two-component 

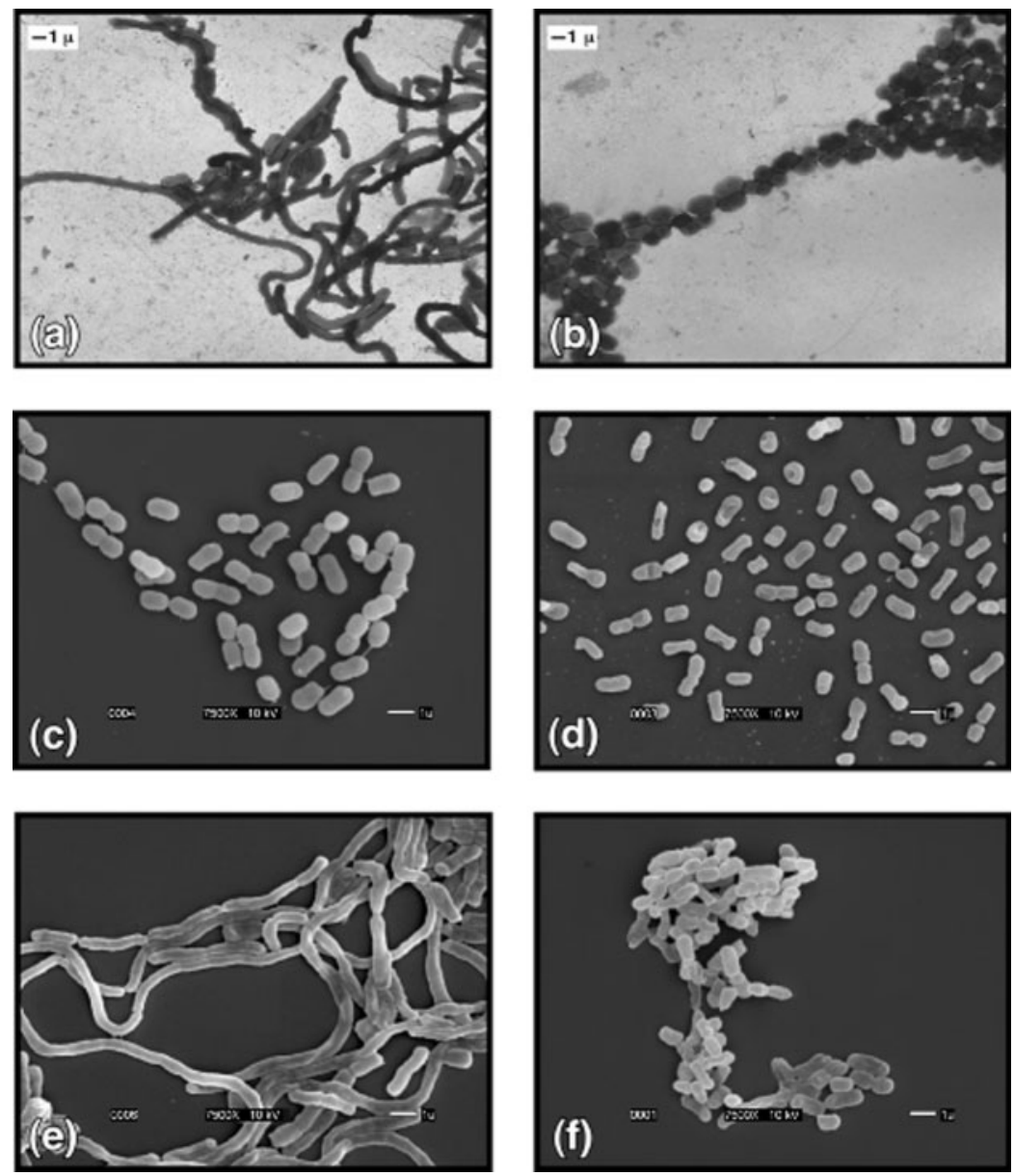

Fig. 6. TEM of $B f m 17$ (a) and Bfm17 cells harbouring pMU300 (b) cultured on Tris-M9 agar and stained with ammonium molybdate after lifting onto carbon-coated grids. SEM of parental (c, d) and Bfm17 (e, f) cells either suspended in the planktonic phase (c, e) or attached to the side of a square polystyrene Petri dish $(d, f)$. All cells were cultured in TrisM9 broth. regulatory systems such as CpxRA in Gram-negative bacteria. Mutations that produce hyperactive CpxR derivatives alter the distribution and assembly of the FtsZ protein in the cell (Pogliano et al., 1998). The random distribution of this cell division protein results in abnormal septation and cell division and, consequently, in the emergence of bacterial cells with aberrant morphology. This latter observation is of particular significance to our work because it shows that a particular two-component regulatory system, such as CpxRA, can control the expression of central cellular processes involved in cell division and morphology as well as in the assembly of surface-exposed appendices and structures. The two latter cellular components include somatic pili, such as the $\mathrm{P}$ pili of uropathogenic E. coli (Hung et al., 2001), and curli, a particular class of pili produced by E. coli K-12 (Dorel et al., 1999), both of which are required for cell attachment to solid surfaces and the development of biofilms.

\section{ACKNOWLEDGEMENTS}

Funds from Miami University and National Science Foundation (NSF) grant 0420479 supported part of this work. We thank Richard Edelmann, Matt Duley and the Miami University Electron Microscopy Facility for their help with electron microscopy. We are grateful to C. Wood, coordinator of the Miami University Center of Bioinformatics and Functional Genomics, for his support and assistance with automated DNA sequencing and nucleotide sequence analysis.

\section{REFERENCES}

Actis, L. A., Potter, S. A. \& Crosa, J. H. (1985). Iron-regulated outer membrane protein OM2 of Vibrio anguillarum is encoded by virulence plasmid pJM1. J Bacteriol 161, 736-742.

Actis, L. A., Tolmasky, M. E., Crosa, L. M. \& Crosa, J. H. (1995). Characterization and regulation of the expression of FatB, an iron transport protein encoded by the pJM1 virulence plasmid. Mol Microbiol 17, 197-204.

Barcak, G. J., Chandler, M. S., Redfield, R. J. \& Tomb, J. F. (1991). Genetic systems in Haemophilus influenzae. Methods Enzymol 204, 321-342.

Bergogne-Berezin, E. (2001). The increasing role of Acinetobacter species as nosocomial pathogens. Curr Infect Dis Rep 3, 440-444.

Bourret, R. B. \& Stock, A. M. (2002). Molecular information processing: lessons from bacterial chemotaxis. J Biol Chem 277, 9625-9628.

Bouvet, P. J. M. \& Grimont, P. A. D. (1986). Taxonomy of the genus Acinetobacter with the recognition of Acinetobacter baumannii sp. nov., Acinetobacter haemolyticus sp. nov., Acinetobacter johnsonii sp. nov., and Acinetobacter junii sp. nov. and emended descriptions of 
Acinetobacter calcoaceticus and Acinetobacter lwoffii. Int J Syst Bacteriol 36, 228-240.

Bradford, M. (1976). A rapid and sensitive method for the quantitation of microgram quantities of proteins utilizing the principle of protein-dye binding. Anal Biochem 72, 249-252.

Dahl, M. K., Msadek, T., Kunst, F. \& Rapoport, G. (1992). The phosphorylation state of the $\mathrm{DegU}$ response regulator acts as a molecular switch allowing either degradative enzyme synthesis or expression of genetic competence in Bacillus subtilis. J Biol Chem 267, 14509-14514.

Davis, K. A., Moran, K. A., McAllister, C. K. \& Gray, P. J. (2005). Multidrug-resistant Acinetobacter extremity infections in soldiers. Emerg Infect Dis 11, 1218-1224.

Dijkshoorn, L., Nemec, A. \& Seifert, H. (2007). An increasing threat in hospitals: multidrug-resistant Acinetobacter baumannii. Nat Rev Microbiol 5, 939-951.

Dorel, C., Vidal, O., Prigent-Combaret, C., Vallet, I. \& Lejeune, P. (1999). Involvement of the Cpx signal transduction pathway of E. coli in biofilm formation. FEMS Microbiol Lett 178, 169-175.

Dorsey, C. W., Tomaras, A. P. \& Actis, L. A. (2002). Genetic and phenotypic analysis of Acinetobacter baumannii insertion derivatives generated with a transposome system. Appl Environ Microbiol 68, 6353-6360.

Dorsey, C. W., Tolmasky, M. E., Crosa, J. H. \& Actis, L. A. (2003). Genetic organization of an Acinetobacter baumannii chromosomal region harbouring genes related to siderophore biosynthesis and transport. Microbiology 149, 1227-1238.

Dorsey, C. W., Laarakker, M. C., Humphries, A. D., Weening, E. H. \& Baumler, A. J. (2005). Salmonella enterica serotype Typhimurium MisL is an intestinal colonization factor that binds fibronectin. Mol Microbiol 57, 196-211.

Feinberg, A. P. \& Vogelstein, B. (1983). A technique for radiolabeling DNA restriction endonuclease fragments to high specific activity. Anal Biochem 132, 6-13.

Fukuchi, K., Kasahara, Y., Asai, K., Kobayashi, K., Moriya, S. \& Ogasawara, N. (2000). The essential two-component regulatory system encoded by $y y c F$ and $y y c G$ modulates expression of the fts $A Z$ operon in Bacillus subtilis. Microbiology 146, 1573-1583.

Howell, A., Dubrac, S., Noone, D., Varughese, K. I. \& Devine, K. (2006). Interactions between the YycFG and PhoPR two-component systems in Bacillus subtilis: the PhoR kinase phosphorylates the noncognate YycF response regulator upon phosphate limitation. Mol Microbiol 59, 1199-1215.

Hung, D. L., Raivio, T. L., Jones, C. H., Silhavy, T. J. \& Hultgren, S. J. (2001). Cpx signaling pathway monitors biogenesis and affects assembly and expression of P pili. EMBO J 20, 1508-1518.

Hunger, M., Schmucker, R., Kishan, V. \& Hillen, W. (1990). Analysis and nucleotide sequence of an origin of DNA replication in Acinetobacter calcoaceticus and its use for Escherichia coli shuttle plasmids. Gene 87, 45-51.

Jiang, M., Shao, W., Perego, M. \& Hoch, J. A. (2000). Multiple histidine kinases regulate entry into stationary phase and sporulation in Bacillus subtilis. Mol Microbiol 38, 535-542.

Joly-Guillou, M. L. (2005). Clinical impact and pathogenicity of Acinetobacter. Clin Microbiol Infect 11, 868-873.

Loehfelm, T. W., Luke, N. R. \& Campagnari, A. A. (2008). Identification and characterization of an Acinetobacter baumannii biofilm-associated protein. J Bacteriol 190, 1036-1044.

Martinez-Hackert, E. \& Stock, A. M. (1997). Structural relationships in the OmpR family of winged-helix transcription factors. J Mol Biol 269, 301-312.
Meade, H. M., Long, S. R., Ruvkum, G. B., Brown, S. E. \& Ausubel, F. M. (1982). Physical and genetic characterization of symbiotic and auxotrophic mutants of Rhizobium meliloti induced by transposon Tn5 mutagenesis. J Bacteriol 149, 114-122.

Mok, K. C., Wingreen, N. S. \& Bassler, B. L. (2003). Vibrio harveyi quorum sensing: a coincidence detector for two autoinducers controls gene expression. EMBO J 22, 870-881.

Ng, W. L., Robertson, G. T., Kazmierczak, K. M., Zhao, J., Gilmour, R. \& Winkler, M. E. (2003). Constitutive expression of PcsB suppresses the requirement for the essential VicR $(\mathrm{YycF})$ response regulator in Streptococcus pneumoniae R6. Mol Microbiol 50, 1647-1663.

O'Toole, G. A. \& Kolter, R. (1998). Initiation of biofilm formation in Pseudomonas fluorescens WCS365 proceeds via multiple, convergent signalling pathways: a genetic analysis. Mol Microbiol 28, 449-461.

O'Toole, G. A., Gibbs, K. A., Hager, P. W., Phibbs, P. V., Jr \& Kolter, R. (2000). The global carbon metabolism regulator Crc is a component of a signal transduction pathway required for biofilm development by Pseudomonas aeruginosa. J Bacteriol 182, 425-431.

Pao, G. M. \& Saier, M. H., Jr (1995). Response regulators of bacterial signal transduction systems: selective domain shuffling during evolution. J Mol Evol 40, 136-154.

Pogliano, J., Dong, J. M., De Wulf, P., Furlong, D., Boyd, D., Losick, R., Pogliano, K. \& Lin, E. C. (1998). Aberrant cell division and random FtsZ ring positioning in Escherichia coli $c p x A^{*}$ mutants. J Bacteriol 180, 3486-3490.

Sambrook, J. \& Russell, D. W. (2001). Molecular Cloning: a Laboratory Manual, 3rd edn. Cold Spring Harbor, NY: Cold Spring Harbor Laboratory.

Schembri, M. A., Kjaergaard, K. \& Klemm, P. (2003). Global gene expression in Escherichia coli biofilms. Mol Microbiol 48, 253-267.

Shine, J. \& Dalgarno, L. (1974). The 3 '-terminal sequence of Escherichia coli $16 \mathrm{~S}$ ribosomal RNA: complementary to nonsense triplets and ribosome binding sites. Proc Natl Acad Sci U S A 71, $1342-1346$.

Stanley, N. R. \& Lazazzera, B. A. (2004). Environmental signals and regulatory pathways that influence biofilm formation. Mol Microbiol 52, 917-924.

Stock, A. M., Robinson, V. L. \& Goudreau, P. N. (2000). Twocomponent signal transduction. Annu Rev Biochem 69, 183-215.

Studier, F. W., Rosenberg, A. H., Dunn, J. J. \& Dubendorff, J. W. (1990). Use of T7 RNA polymerase to direct expression of cloned genes. Methods Enzymol 185, 60-89.

Tomaras, A. P., Dorsey, C. W., Edelmann, R. E. \& Actis, L. A. (2003). Attachment to and biofilm formation on abiotic surfaces by Acinetobacter baumannii: involvement of a novel chaperone-usher pili assembly system. Microbiology 149, 3473-3484.

Towbin, H., Staehelin, T. \& Gordon, J. (1979). Electrophoretic transfer of proteins from polyacrylamide gel to nitrocellulose sheets: procedure and some applications. Proc Natl Acad Sci U S A 76, 4350-4354.

Vallenet, D., Nordmann, P., Barbe, V., Poirel, L., Mangenot, S., Bataille, E., Dossat, C., Gas, S., Kreimeyer, A. \& other authors (2008). Comparative analysis of Acinetobacters: three genomes for three lifestyles. PLoS ONE 3, e1805.

Vidal, R., Dominguez, M., Urrutia, H., Bello, H., Gonzalez, G., Garcia, A. \& Zemelman, R. (1996). Biofilm formation by Acinetobacter baumannii. Microbios 86, 49-58.

Vidal, R., Dominguez, M., Urrutia, H., Bello, H., Garcia, A., Gonzalez, G. \& Zemelman, R. (1997). Effect of imipenem and sulbactam on sessile cells of Acinetobacter baumannii growing in biofilm. Microbios 91, 79-87. 
Vidal, O., Longin, R., Prigent-Combaret, C., Dorel, C., Hooreman, M. \& Lejeune, P. (1998). Isolation of an Escherichia coli K-12 mutant strain able to form biofilms on inert surfaces: involvement of a new ompR allele that increases curli expression. J Bacteriol 180, 2442-2449.

Villegas, M. V. \& Hartstein, A. I. (2003). Acinetobacter outbreaks, 1977-2000. Infect Control Hosp Epidemiol 24, 284-295.

Villers, D., Espaze, E., Coste-Burel, M., Giauffret, F., Ninin, E., Nicolas, F. \& Richet, H. (1998). Nosocomial Acinetobacter baumannii infections: microbiological and clinical epidemiology. Ann Intern Med 129, 182-189.

Wanner, B. L. (1992). Is cross regulation by phosphorylation of two-component response regulator proteins important in bacteria? J Bacteriol 174, 2053-2058.
Wu, C.-J. \& Janssen, G. R. (1997). Expression of a streptomycete leaderless mRNA encoding chloramphenicol acetyltransferase in Escherichia coli. J Bacteriol 179, 6824-6830.

Yanisch-Perron, C., Vieira, J. \& Messing, J. (1985). Improved M13 phage cloning vectors and host strains: nucleotide sequence of the M13mp18 and pUC19 vectors. Gene 33, 103-109.

Yildiz, F. H., Dolganov, N. A. \& Schoolnik, G. K. (2001). VpsR, a member of the response regulators of the two-component regulatory systems, is required for expression of $v p s$ biosynthesis genes and EPS(ETr)-associated phenotypes in Vibrio cholerae O1 El Tor. J Bacteriol 183, 1716-1726.

Edited by: J. G. Shaw 